\title{
AN INVERSION THEOREM FOR HANKEL TRANSFORMS ${ }^{1}$
}

ALAN L. SCHWARTZ ${ }^{2}$

It is a well-known fact of classical Fourier analysis that if $f$ is a function integrable on the real line and of bounded variation in a neighborhood of $x$, then

$$
\lim _{\lambda \rightarrow \infty} \int_{-\lambda}^{\lambda} e^{i u x} d u \int_{-\infty}^{\infty} f(y) e^{-i u y} d y=(1 / 2)\{f(x+0)+f(x-0)\} .
$$

Analogous results hold for other integral transforms. It is our intention to study the behaviour of a similar inversion formula for the Hankel transforms defined below.

Let $\nu$ be a fixed real number exceeding $(-1 / 2)$ and let $L$ consist of all functions measurable on $0<x<\infty$ such that

$$
\|f\|=\int_{0}^{\infty}|f(x)| d m(x)<\infty
$$

where

$$
d m(x)=\left[2^{\nu} \Gamma(\nu+1)\right]^{-1} x^{2 \nu+1} d x
$$

Let

$$
\partial(x)=2^{\nu} \Gamma(\nu+1) x^{-\nu} J_{\nu}(x),
$$

where $J_{\nu}$ is the Bessel function of the first kind of order $\nu$. We are interested in whether the following formula analogous to (1) holds

$$
\begin{aligned}
\lim _{\lambda \rightarrow \infty} \int_{0}^{\lambda} \mathfrak{J}(x u) d m(u) \int_{0}^{\infty} f(y) \mathfrak{J}(u y) d m(y) & \\
& =(1 / 2)\{f(x+0)+f(x-0)\} .
\end{aligned}
$$

Equation (2) does not hold under circumstances as general as those for which (1) holds. What is needed is a restriction on the behaviour of $f$ near 0 ; we shall prove the following theorem after a few remarks.

TheOREM. Suppose $f$ is in $L$ and

Received by the editors December 27, 1968.

${ }^{1}$ Part of the work in this paper was included in the author's doctoral dissertation, which was written at the University of Wisconsin.

${ }^{2}$ This research was supported in part by the National Science Foundation, contract number GP-6764. 


$$
\int_{0}^{1}|f(y)| y^{\nu+(1 / 2)} d y<\infty,
$$

then if $x>0$ and if $f$ is of bounded variation in a neighborhood of $x$, (2) holds.

Finally we will show by means of an example that the exponent $\nu+(1 / 2)$ in (3) cannot be increased.

We will need the following well-known properties of Bessel functions

$$
J_{\mu}(x)=(2 / \pi x)^{(1 / 2)} \cos (x-(1 / 2) \mu \pi-(1 / 4) \pi)+O\left(x^{-(3 / 2)}\right)
$$

as $x \rightarrow \infty$,

in particular

$$
\begin{aligned}
& J_{\mu}(x)=O(1 / \sqrt{ } x) \quad \text { as } x \rightarrow \infty, \\
& J_{\mu}(x)=O\left(x^{\mu}\right) \quad \text { as } x \rightarrow 0 \text {, } \\
& \int_{0}^{\lambda} J_{\mu}(u y) J_{\mu}(u x) u d u \\
& =\lambda\left(x^{2}-y^{2}\right)^{-1}\left\{x J_{\nu+1}(\lambda x) J_{\nu}(\lambda y)-y J_{\nu+1}(\lambda y) J_{\nu}(\lambda x)\right\}
\end{aligned}
$$

(see [5 p. 134]).

Proof of The Theorem. The integral in (2) can be written as a sum of two integrals with $y$ in the ranges $(0, \delta)$ and $(\delta, \infty)$ where $0<\delta<x$. The proof of Hankel's theorem in [4, pp. 240-242] is easily adapted to show

$$
\lim _{\lambda \rightarrow \infty} \int_{0}^{\lambda} \mathcal{J}(x u) d m(u) \int_{\delta}^{\infty} f(y) \mathcal{J}(u y) d m(y)=(1 / 2)\{f(x+0)+f(x-0)\}
$$

condition (3) does not enter that argument. Thus it suffices to show

$$
\lim _{\lambda \rightarrow \infty} \int_{0}^{\lambda} g(x u) d m(u) \int_{0}^{\delta} f(y) \mathcal{J}(u y) d m(y)=0 .
$$

By Fubini's theorem and (7) this last integral is equal to

$$
\begin{aligned}
\lambda x^{1-\nu} J_{\nu+1}(\lambda x) \int_{0}^{\delta} f(y)\left(x^{2}-y^{2}\right)^{-1} y^{\nu+1} J_{\nu}(\lambda y) d y & \\
& -\lambda x^{-\nu} J_{\nu}(\lambda x) \int_{0}^{\delta} f(y)\left(x^{2}-y^{2}\right)^{-1} y^{\nu+2} J_{\nu}(\lambda y) d y .
\end{aligned}
$$

We will show that the first integral is $o(1 / \sqrt{ } \lambda)$ as $\lambda \rightarrow \infty$. The same 
methods serve to analyze the second integral and the theorem will be proved.

From (6) we have

$$
\begin{aligned}
\left|\int_{0}^{(1 / \lambda)} f(y)\left(x^{2}-y^{2}\right)^{-1} J_{\nu}(\lambda y) y^{\nu+1} d y\right| \\
=O\left(\lambda^{\nu}\right) \int_{0}^{(1 / \lambda)}|f(y)| y^{2 \nu+1} d y \\
=O(1 / \sqrt{ } \lambda) \int_{0}^{(1 / \lambda)}|f(y)| y^{\nu+(1 / 2)} d y=o(1 / \sqrt{ } \lambda)
\end{aligned}
$$

by (3). For $y \geqq(1 / \lambda)$ we use the asymptotic expansion (4). The cosine term contributes

$$
\begin{array}{r}
\left(\frac{2}{\pi \lambda}\right)^{(1 / 2)} \int_{(1 / \lambda)}^{\delta} f(y)\left(x^{2}-y^{2}\right)^{-1} \cos (\lambda y-(1 / 2) \nu \pi-(1 / 4) \pi) y^{\nu+(1 / 2)} d y \\
=o(1 / \sqrt{ } \lambda)
\end{array}
$$

by the classical Riemann-Lebesgue lemma. Finally the $O$ term is estimated in two parts:

$$
\begin{aligned}
\left|\int_{(1 / \lambda)}^{(1 / \sqrt{ } \lambda)} f(y)\left(x^{2}-y^{2}\right)^{-1} O\left((\lambda y)^{-(3 / 2)}\right) y^{\nu+1} d y\right| \\
=O(1 / \sqrt{ } \lambda) \int_{(1 / \lambda)}^{(1 / \sqrt{ } \lambda)}|f(y)|(\lambda y)^{-1} y^{\nu+(1 / 2)} d y \\
=O(1 / \sqrt{ } \lambda) \int_{(1 / \lambda)}^{(1 / \sqrt{ } \lambda)}|f(y)| y^{\nu+(1 / 2)} d y=o(1 / \sqrt{ } \lambda)
\end{aligned}
$$

by (3); and

$$
\begin{aligned}
& \left|\int_{(1 / \sqrt{ } \lambda)}^{\delta} f(y)\left(x^{2}-y^{2}\right)^{-1} O\left((\lambda y)^{-(3 / 2)}\right) y^{\nu+1} d y\right| \\
& \quad=O(1 / \lambda) \int_{(1 / \sqrt{ } \lambda)}^{\delta}|f(y)|(y \sqrt{ } \lambda)^{-1} y^{\nu+(1 / 2)} d y=O(1 / \lambda)=o(1 / \sqrt{ } \lambda)
\end{aligned}
$$

by (3). Q.E.D.

We now give an example that shows that the exponent $\nu+(1 / 2)$ in (3) cannot be increased. Let

$$
\begin{array}{rlrl}
f(y) & =y^{-(v+(3 / 2))} & 0<y & \leqq 1 \\
& =0 & y & >1
\end{array}
$$


and let $x>1$. Let

$$
I_{\lambda}=\int_{0}^{\lambda} g(x u) d m(u) \int_{0}^{\infty} f(y) g(u y) d m(y) .
$$

Thus (2) holds if and only if

$$
I_{\lambda} \rightarrow 0 \quad \text { as } \lambda \rightarrow \infty .
$$

We will find a sequence $\lambda_{i}$ such that

$$
\lambda_{i} \rightarrow \infty \quad \text { as } i \rightarrow \infty,
$$

and such that for some positive constant $C$

$$
\left|I_{\lambda_{i}}\right|>C \quad(i=1,2,3, \cdots) .
$$

Let $x_{1}, x_{2}, x_{3}, \cdots$ be the positive real zeros of $J_{v}(x)$ in ascending order and let $\lambda_{i}=x_{i} / x$. Then by (7) and Fubini's theorem

$$
\begin{aligned}
I_{\lambda_{i}} & =\lambda_{i} x^{1-\nu} J_{\nu+1}\left(x_{i}\right) \int_{0}^{1} \frac{J_{\nu}\left(\lambda_{i} y\right)}{\left(x^{2}-y^{2}\right) y^{1 / 2}} d y, \\
& =x^{1-\nu} \lambda_{i}{ }^{1 / 2} J_{\nu+1}\left(x_{i}\right) \int_{0}^{\lambda_{i}} \frac{J_{\nu}(w)}{\left[x^{2}-\left(w / \lambda_{i}\right)^{2}\right] w^{1 / 2}} d w .
\end{aligned}
$$

It can be easily shown that

$$
\lim _{i \rightarrow \infty} \int_{0}^{\lambda_{i}} \frac{J_{\nu}(w) d w}{\left[x^{2}-\left(w / \lambda_{i}\right)^{2}\right] w^{1 / 2}}=x^{-2} \int_{0}^{\infty} J_{\nu}(w) w^{-1 / 2} d w .
$$

This last expression has the value

$$
\Gamma((2 \nu+1) / 4) / 2^{1 / 2} \Gamma((2 \nu+3) / 4)
$$

(see $[2$, p. 22, formula (7)]).

From (4) it follows that

$$
\begin{aligned}
J_{\nu+1}(x) & =(2 / \pi x)^{1 / 2} \cos (x-\beta-(\pi / 2))+O\left(x^{-3 / 2}\right) \\
& =(2 / \pi x)^{1 / 2} \sin (x-\beta)+O\left(x^{-3 / 2}\right)
\end{aligned}
$$

where $\beta=(2 \nu+1) \pi / 4$ and

$$
J_{\nu}(x)=(2 / \pi x)^{1 / 2} \cos (x-\beta)+O\left(x^{-3 / 2}\right) .
$$

Since $J_{\nu}\left(x_{i}\right)=0$, we see that

$$
\left|J_{\nu+1}\left(x_{i}\right)\right| \geqq\left(\pi x_{i}\right)^{-1 / 2}
$$

for $i$ sufficiently large. Thus for some constant $C$ we have 


$$
x^{1-\nu} \lambda_{i}^{1 / 2}\left|J_{\nu+1}\left(x_{i}\right)\right| \geqq C
$$

and so

$$
\left|I_{\lambda_{i}}\right| \geqq x^{\nu-1} C .
$$

Thus, it follows that $\nu+(1 / 2)$ is indeed the largest exponent in (3) for which the theorem holds.

REMARK. Suppose $\nu=(n-2) / 2$ where $n$ is an integer greater than 1 , and suppose $f$ is defined on $R_{n}$, Euclidean $n$-space. $f$ is radial if there is a function $g$ defined on $(0, \infty)$ such that

$$
f(\mathbf{x})=g(|\mathbf{x}|)
$$

for almost all $\mathrm{x}$ in $R_{n}$. Then $f$ is integrable if and only if $g$ is in $L$; furthermore [1, p. 69] $\int_{0}^{\infty} g(y) \mathcal{J}(u y) d m(u)$ is essentially the Fourier transform of $f$ at $y$ for any point $y$ such that $|y|=u$. Then our theorem says that the multiple Fourier transform of $f$ can be inverted by spherical sums if $f(x)|x|^{(n-1) / 2}$ is integrable and our example yields one of a function supported on $|x| \leqq 1$ for which localization fails to hold for the spherical sums.

The author wishes to express his deep thanks to Professors Rudin and Askey of the University of Wisconsin.

\section{REFERENCES}

1. S. Bochner and K. Chandrasekharan, Fourier transforms, Princeton Univ. Press, Princeton, N. J., 1949.

2. Erdélyi et al, Tables of integral transforms, Vol. II, McGraw-Hill, New York, 1953.

3. I. I. Hirschman, Jr., Variation diminishing Hankel transforms, J. Analyse Math. 8 (1960/1961), 307-336.

4. E. C. Titchmarsh, Introduction to the theory of Fourier integrals, Oxford Univ. Press, London, 1959.

5. G. N. Watson, A treatise on the theory of Bessel functions, Cambridge Univ. Press, London, 1966.

UNIVERSITY OF MISSOURI 